\title{
Vascular biology and bone formation: hints from HIF
}

\author{
Dwight A. Towler \\ Department of Medicine, Center for Cardiovascular Research, Division of Bone and Mineral Diseases, \\ Washington University School of Medicine, St. Louis, Missouri, USA.
}

\begin{abstract}
In this issue of the JCI, Wang, Clemens, and colleagues demonstrate that hypoxia-inducible factor $\alpha(\mathrm{HIF} \alpha)$ signaling in bone-building osteoblasts is central to the coupling of angiogenesis and long bone development in mice (see the related article beginning on page 1616). They show that bone formation controlled by osteoblast HIF $\alpha$ signaling is not cell autonomous but is coupled to skeletal angiogenesis dependent upon VEGF signaling. Thus, strategies that promote HIF $\alpha$ signaling in osteoblasts may augment bone formation and accelerate fracture repair.
\end{abstract}

Tremendous unmet clinical needs exist in musculoskeletal medicine. The direct costs of musculoskeletal diseases in the Unites States are well over $\$ 100$ billion per annum (1). Osteoporosis and osteoarthritis are recognized as common and clinically important, but other serious skeletal diseases also afflict our populace. In the setting of type 2 diabetes mellitus (T2DM), lower-extremity musculoskeletal disease is prevalent, costly, and exceedingly difficult to manage, with fracture, arthropathy, ischemia, ulcer, and infection commonly confronting patients and clinicians. The total costs associated with lower-extremity amputation in T2DM alone are greater than the combined costs of treating fatal and nonfatal myocardial infarction associated with T2DM (2). Stage-specific and disease-specific strategies are necessary to safely promote bone formation in individuals with: (a) underlying vasculopathies, such as those associated with diabetes or renal failure; (b) underlying malignancy of any sort; (c) extant osteoporosis that has removed trabecular templates for bone apposition; (d)

Nonstandard abbreviations used: BMP, bone morphogenetic protein; BV/TV, bone volume/tissue volume; HIF, hypoxia-inducible factor; $\Delta$ Hifla mice, mice with conditional deletion of Hif1a in osteoblasts; PHD, prolyl hydroxylase; pVHL, von Hippel-Lindau protein; SM22, smooth muscle $22 \mathrm{kDa}$; T2DM, type 2 diabetes mellitus; TNFR2, TNF receptor $2 ; \Delta V h l$ mice, mice with conditional deletion of $\mathrm{Vbl}$ in osteoblasts; $\Delta \mathrm{Vbl} / \Delta \mathrm{Hifla}$ mice, mice with conditional deletion of both $\mathrm{Vhl}$ and Hifla in osteoblasts.

Conflict of interest: The author receives grant support from the NIH and the Barnes-Jewish Hospital Foundation and receives compensation as an ad hoc consultant for Novartis, Lilly, and GlaxoSmithKline.

Citation for this article: J. Clin. Invest. 117:1477-1480 (2007). doi:10.1172/JCI32518. osteoporosis in the setting of childhood growth and open epiphyses; and (e) drugor coagulopathy-related disorders that cause avascular necrosis.

In this issue of the JCI, the study by Wang et al. (3) affords us a better understanding of the mechanisms coupling bone and vascular physiology, providing insights useful for devising novel strategies to address the mounting unmet needs in orthopedic medicine. The vasculature provides: (a) a sustentacular niche and source of adult mesenchymal stem cells, including osteoprogenitors; (b) the organizational and rate-limiting "point of reference" for Haversian bone formation; and (c) the conduit for calcium, phosphate, hematopoietic, and nutrient supply necessary for mineralization and calcium mobilization (4-11). We know precious little about how the vasculature integrates and conveys signals during skeletogenesis. However, VEGF (9) has emerged as the prototypic osteogenic-angiogenic coupling factor (10). The $V E G F$ gene encodes a secreted polypeptide globally required for vasculogenesis and angiogenesis (11). Bioactivity is modulated by VEGF gene expression - and differential splicing that generates three unique gene products - with signals transduced via specific VEGF receptor tyrosine kinases (9). The name "VEGF" belies its contributions to osteoblast ontogeny, chondrocyte physiology, and osteoclast formation (10). $V E G F$ signaling is tightly regulated in bone, coupled to morphogenetic, metabolic, inflammatory, and mechanical cues that control mineral metabolism. In addition to regulating the expansion and survival of mesenchymal progenitors (Figure 1 and below), signals provided by VEGFR2 mediate angiogenic cross-talk with TNF receptor 2 (TNFR2) (12), critical for postnatal defense against limb ischemia (13). Given the contributions of bone-vascular interactions to all aspects of bone biology, our limited understanding of this important physiology impedes development of novel bone anabolic therapies.

\section{New hints from HIF}

In their current study, Wang, Clemens, and colleagues (3) significantly advance our understanding of bone-vascular coupling by establishing the critical role for osteoblast hypoxia-inducible factor $1 \alpha$ (HIF- $1 \alpha$ ) and HIF- $2 \alpha$ in bone formation (Figure 1). As recently reviewed (14), HIFs are components of heterodimeric, hypoxia-activated transcription factor complexes that bind to well-characterized DNA cognates called hypoxia-response elements, activating the expression of genes such as VEGF and erythropoietin that can improve tissue oxygen delivery (15). Dependent upon cellular context, HIF- $1 \alpha$ and HIF- $2 \alpha$ may or may not exhibit functional redundancy (14). The Clemens group first showed that osteoblasts express all necessary components of the oxygen-sensing pathway in addition to HIF- $1 \alpha$ and HIF-2 $\alpha$ (herein collectively referred to as HIF $\alpha$ ). These components include the oxygen-dependent prolyl hydroxylases (PHDs) that "tag" $\mathrm{HIF} \alpha$ for recognition by the E3 ubiquitin ligase von Hippel-Lindau protein (pVHL) and pVHL itself, necessary for HIF $\alpha$ proteosomal degradation (3) (Figure 1). Moreover, the authors demonstrate hypoxiadependent nuclear accumulation of HIF $\alpha$ and concomitant upregulation of VEGF expression, indicating intact oxygen-sensing functions in osteoblasts. To evaluate the biological importance of osteoblast HIF signaling in vivo, the authors used Cre-lox technology; they implemented the bone-specific human osteocalcin promoter as a delivery module for Cre recombinase expression in mice possessing floxed $\mathrm{Vhl}$ 


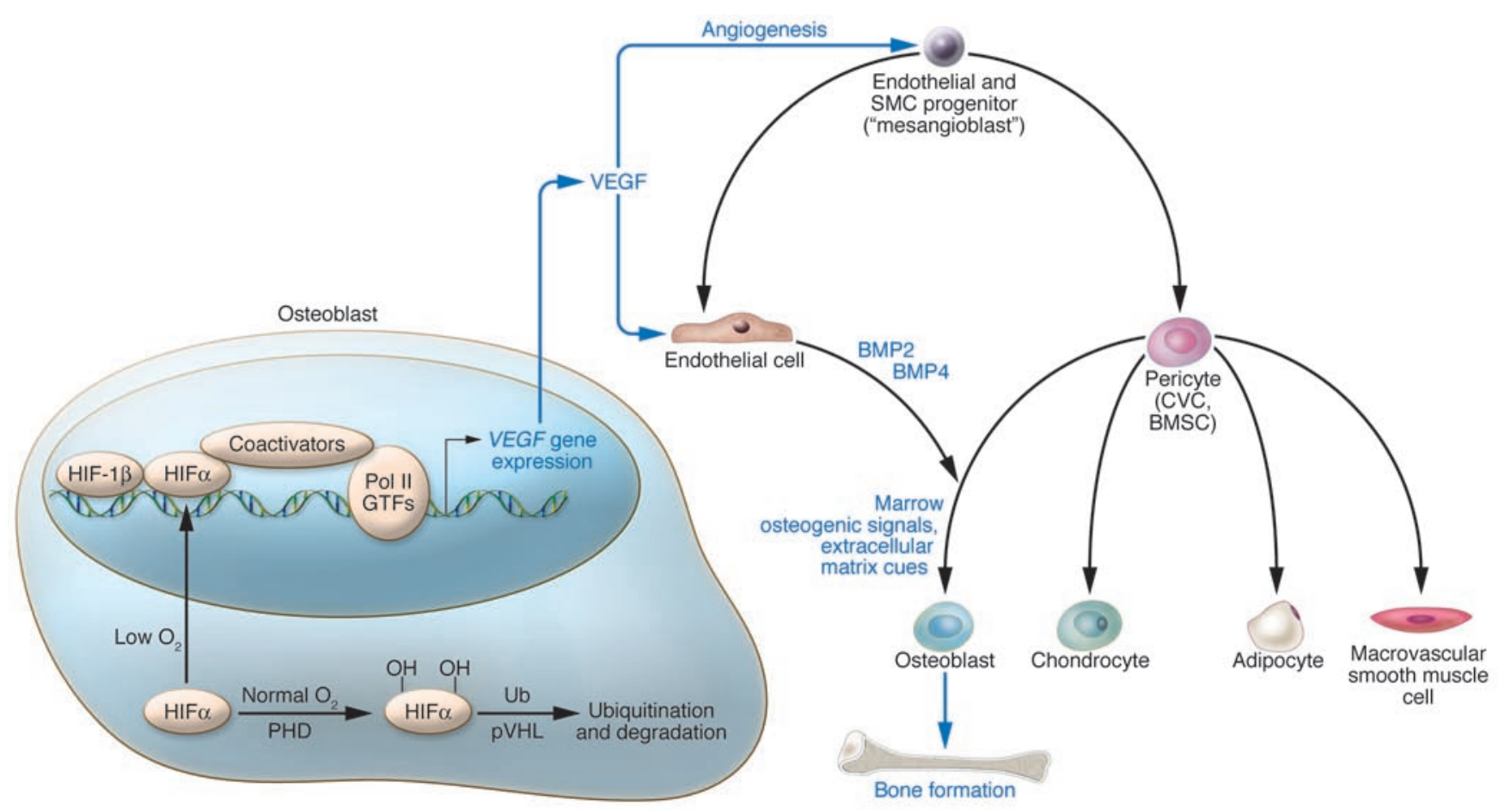

Figure 1

Working model of osteogenic-angiogenic coupling in trabecular bone. Recent data from multiple laboratories (16-24) have indicated that microvascular smooth muscle cells known as pericytes represent osteoprogenitors capable of bone formation when placed in the correct microenvironment. Pericytes appear to arise from a vessel-associated stem cell progenitor (mesoangioblast; refs. 20, 31, 32), and during the process of mesoderm growth and angiogenesis, this VEGFR2-expressing stem cell undergoes expansion $(23,31)$. In this issue of the $\mathrm{JCl}$, Wang et al. (3) demonstrate that osteoblast HIF $\alpha$ subunits, transcriptional regulators of VEGF expression, represent rate-limiting components of osteogenicangiogenic coupling and trabecular bone formation. Augmentation of osteoblast HIF $\alpha$ expression and bone formation was achieved by conditionally deleting $\mathrm{Vhl}$, the gene encoding $\mathrm{pVHL}$ — the E3 ubiquitin ligase necessary for HIF $\alpha$ degradation. Bone formation was not cell autonomous - i.e., not dependent solely on osteoblast functions - but required VEGF-mediated paracrine signals in bone that stimulated angiogenesis. Since VEGF can expand VEGFR2-expressing mesoangioblast numbers during angiogenesis (33), this process may drive the increase in osteoblast numbers that promotes massive trabecular bone formation in the osteogenic marrow environment. PHD enzyme activity is also required for HIF $\alpha$ degradation, oxidatively "tagging" HIF $\alpha$ for recognition by $\mathrm{pVHL}$. In addition to low oxygen levels (as shown here), mechanical stimuli, TNF- $\alpha$, and reactive oxygen species can also upregulate HIF $\alpha$ expression (29). Strategies that augment osteoblast HIF $\alpha / V E G F$ signaling by selectively inhibiting skeletal PHD may increase bone formation and enhance fracture healing. BMSC, bone marrow stromal cell; CVC, calcifying vascular cell; GTF, general transcription factor; Pol II, RNA polymerase II; Ub, ubiquitin.

alleles. This strategy abrogates pVHL accumulation in mature osteoblasts (3). In this conditional knockout, denoted $\Delta V h l$, osteoblast accumulation of both HIF- $1 \alpha$ and HIF- $2 \alpha$ was induced due to the absence of pVHL-dependent degradation (Figure 1). The in vivo effects on long bone formation were remarkable; bone volume/tissue volume (BV/TV) increased by $70 \%$ with osteoblast-specific induction of HIF $\alpha$ subunits. Detailed histomorphometry revealed very early postnatal increases in trabecular osteoblast numbers during long bone modeling - numbers that "normalized" once a new steady state of high bone mass was achieved in $\Delta V b l$ mice (3). In culture, osteoblasts possessing floxed $\mathrm{Vhl}$ alleles also upregulated HIF $\alpha$ when transduced with an adenoviral Cre vector - with concomitant induction of VEGF. HIF $\alpha$ induction did not alter cultured osteoblast proliferation rate, apoptotic rate, matrix synthetic activity, or mineral deposition ex vivo; thus, the bone anabolic actions of osteoblast HIF $\alpha$ induction observed in vivo were not cell autonomous. Bone histomorphometry and serum biochemistries showed no decrements in bone-resorbing osteoclast numbers or activity, excluding overt contributions of osteoclast insufficiency to increased BV/TV in $\Delta V b l$ mice. However, a profound increase in bone vascularity was observed for the $\Delta V h l$ mice (3). Moreover, a massive increase in capillary sprouting was exhibited by $\Delta V b l$ long bones maintained in organ culture (3). Angiogenic responses were dependent upon paracrine VEGF actions, since: (a) VEGF-neutralizing antibody abrogated sprouting; and (b) skeletal production of VEGF mRNA was increased in $\Delta V h l$ mice without changes in circulating VEGF. Relationships to HIF $\alpha$-dependent angiogenesis and bone formation were further confirmed using mice in which Hifla was conditionally knocked out in osteoblasts ( $\Delta$ Hifla mice). Bone volume and vascularity were reduced in $\Delta H$ ifla mice, with reductions partially offset by compensatory HIF-2 $\alpha$ expression. The overlapping redundancy of the latter was diligently demonstrated using a third mouse model, $V h l$ gene deletion in $\Delta H i f 1 a$ mice; these $\Delta V b l / \Delta H i f 1 a$ mice exhibited markedly increased HIF- $2 \alpha$ protein accumulation, with concomitant restoration of bone volume and angiogenesis (3). Thus, the authors conclude that osteoblast HIF $\alpha$ signaling is a central component of rate-limiting, osteogenicangiogenic coupling that controls long bone formation. This coupling occurs via 
mechanisms that are not cell autonomous and that utilize paracrine VEGF angiogenic signals to expand osteoblast numbers during long bone development (3).

\section{Angiogenesis and osteoblast ontogeny}

How, then, might the angiogenic response and osteogenic-angiogenic coupling increase osteoblast numbers and provide the massive marrow bone formation observed (3)? Recently, several groups have identified the microvascular smooth muscle cell, the pericyte, as an important osteoprogenitor (16-24) (Figure 1). Demer, Canfield, and colleagues have shown that the pericyte exhibits multipotentiality, capable of osteogenic, chondrogenic, adipogenic, and SMC differentiation $(17,18)$. Molecularly, pericytes express early features of the VSMC lineage, including smooth muscle $22 \mathrm{kDa}(\mathrm{SM} 22), \alpha$-SMC, and species-specific gangliosides demarcated by the $3 \mathrm{G} 5$ monoclonal antibody $(17,19)$. Anatomically, the pericyte is intimately juxtaposed to the endothelial capillary network. In the marrow microenvironment, the bone marrow stromal cell exhibits the histoanatomic characteristics of the pericyte (16). Thus, from this perspective the bone marrow stromal cell - the osteoprogenitor - can be viewed as a tissue-specific pericyte.

What is the ontogeny of the vascular pericyte? Cossu and colleagues provide data suggesting that the mesoangioblast (20), a vessel-associated mesenchymal stem cell with the capacity to differentiate into cells of endothelial and VSMC lineages, might be the source of pericytes. Studies of differentiating murine ES cells confirm the existence of a highly plastic, $\mathrm{VEGFR}^{+}$endothelial-SMC progenitor a mesenchymal stem cell that can give rise to mineralizing osteoblasts in culture via pericytic SMC lineage (23). Skeletal osteoblasts can and do arise from the pericyte cell lineage in vivo; SM22-positive and $\alpha$-SMC-positive cells "coregister" with preosteoblasts identified using Col3.6GFP reporter mice following induction of de novo osteogenesis (24). Thus, concomitant with angiogenic sprouting, VEGF/VEGFR2 signaling is posited to expand the potential osteoprogenitor pool via the pericyte intermediate (Figure 1). However, until better pericyte lineage markers are developed, or the effects of HIF $\alpha$ expansion are tested in the lineage reporter mice (24), the mechanisms proposed remain speculative.

\section{Location, location, location}

Remarkably, unlike in long bone, little if any in vivo effect of osteoblast $V h l$ deletion was observed by Wang et al. in calvarial bone (3). Why might this occur? Unique and differentially regulated ontogeny and angiogenic responses likely contribute (25). There are places in the skeleton (e.g., neural crest-derived calvarial bone, lateral components of the clavicle, the mid-diaphyseal collar of long bone) where bone forms via nonendochondral mechanisms $(11,25)$. Denoted as intramembranous ossification, this osteoblast-mediated mineral deposition occurs directly in the type I collagen-based extracellular matrix - without replacement of a precedent, avascular cartilaginous template by bone and marrow as is required for endochondral ossification $(10,25)$. In the developing skull, it is probable that cranial suture and dural mechanical tension organize angiogenesis necessary for intramembranous ossification (26-28). VEGF expression in osteoblasts is mechanically very responsive (29). Distraction osteogenesis - an orthopedic mechanical manipulation that promotes robust angiogenesis and bone formation via nonendochondral mechanisms - upregulates both HIF- $1 \alpha$ and VEGF (29). Thus, it is tempting to speculate that the differences observed by Wang et al. (3) arise due to differences in the rate-limiting stimuli that control osteogenic-angiogenic coupling in calvarial versus long bone development. For the moment, however, the precise reasons for the differences observed between long bone and calvarial bone formation following $\mathrm{Vhl}$ deletion remain to be determined.

\section{Future directions}

Many questions remain to be answered. Although HIF $\alpha$ clearly regulates osteogenic-angiogenic interactions necessary for bone formation, other secreted molecules in addition to VEGF, such TNF- $\alpha(6,12$, $13)$ and FGF2 $(5,27,28)$, might contribute to this coupling. Potential contributions of paracrine TNF- $\alpha$ signaling - an important activator of TNFR2-VEGFR2 cross-talk $(12,13)$ and bone formation $(6)-$ have yet to be detailed. Should administration of bevacizumab, a clinically useful inhibitory antibody to VEGF (30), be shown to abrogate the bone anabolic effects of osteoblast HIF $\alpha$, this would provide pharmacologic evidence that paracrine VEGF signals are nonredundant in osteogenic-angiogenic coupling. Moreover, since VEGF induces the production of bone morphogenetic pro- tein (BMP) by endothelial cells (5), the consequences of inactivating endothelial BMP expression would help support the evolving working model (Figure 1). The mechanisms that punctuate feed-forward osteogenicangiogenic coupling are not known but clearly exist, since osteoblast numbers and bone formation quickly normalizes postnatally at a higher bone mass (3). The "osteostat" mechanism responsible for this physiologic response will be extremely important to delineate - and may be metabolically as well as mechanically determined. It may be possible to selectively augment skeletal HIF- $1 \alpha$ action - potentially by inhibiting specific PHDs - as one strategy to promote bone formation and fracture healing. It will be important to evaluate how the material and geometric properties of bone manipulated via the HIF $\alpha$ pathway impact bone strength. Finally, better markers are required to unambiguously characterize precursor-product relationships in the mesoangioblast/pericyte/osteoblast lineage; such ontogeny is likely to contribute to changes in trabecular bone mass dependent upon osteoblast HIF $\alpha$ signaling (16, 18). All in all, novel and very important biological principles emerge from the current study (3), i.e., that osteogenesis and angiogenesis are functionally coupled in the marrow microenvironment by osteoblast HIF $\alpha$ signaling. Thus, in addition to osteoblast and osteoclast lineages, the contributions of endothelial cell precursors and their progeny (Figure 1) must be considered in robust studies of bone formation and skeletal homeostasis.

\section{Acknowledgments}

The author is supported by NIH grants HL69229, HL81138, AR43731, and the Barnes-Jewish Hospital Foundation.

Address correspondence to: Dwight A. Towler, Washington University School of Medicine, Barnes-Jewish North Campus Box 8301, 660 South Euclid Avenue, St. Louis, Missouri 63110, USA. Phone: (314) 454-7434; Fax: (314) 454-8434; E-mail: dtowler@im.wustl.edu.

1. Woolf, A.D., and Pflefer, B. 2003. Burden of major musculoskeletal conditions. Bull. World Health Organ. 81:646-656.

2. Clarke, P., Gray, A., Legood, R., Briggs, A., and Holman, R. 2003. The impact of diabetes-related complications on healthcare costs: results from the United Kingdom Prospective Diabetes Study (UKPDS Study No. 65). Diabet. Med. 20:442-450.

3. Wang, Y., et al. 2007. The hypoxia-inducible factor $\alpha$ pathway couples angiogenesis to osteogenesis during skeletal development. J. Clin. Invest. 
117:1616-1626. doi:10.1172/JCI31581.

4. Eghbali-Fatourechi, G.Z., et al. 2005. Circulating osteoblast-lineage cells in humans. N. Engl. J. Med. 352:1959-1966.

5. Bouletreau, P.J., et al. 2002. Hypoxia and VEGF up-regulate BMP-2 mRNA and protein expression in microvascular endothelial cells: implications for fracture healing. Plast. Reconstr. Surg. 109:2384-2397.

6. Gerstenfeld, L.C., et al. 2003. Impaired fracture healing in the absence of TNF-alpha signaling: the role of TNF-alpha in endochondral cartilage resorption. J. Bone Miner. Res. 18:1584-1592.

7. Sorescu, G.P., et al. 2004. Bone morphogenic protein 4 produced in endothelial cells by oscillatory shear stress induces monocyte adhesion by stimulating reactive oxygen species production from a nox1-based NADPH oxidase. Circ. Res. 95:773-779.

8. Csiszar, A., et al. 2006. Bone morphogenetic protein-2 induces proinflammatory endothelial phenotype. Am. J. Pathol. 168:629-638.

9. Ferrara, N. 2004. Vascular endothelial growth factor: basic science and clinical progress. Endocr. Rev. 25:581-611.

10. Zelzer, E., and Olsen, B.R. 2005. Multiple roles of vascular endothelial growth factor (VEGF) in skeletal development, growth, and repair. Curr. Top. Dev. Biol. 65:169-187.

11.Zelzer, E., et al. 2002. Skeletal defects in VEGF(120/120) mice reveal multiple roles for VEGF in skeletogenesis. Development. 129:1893-1904.

12. He, Y., et al. 2006. Critical function of Bmx/Etk in ischemia-mediated arteriogenesis and angiogenesis. J. Clin. Invest. 116:2344-2355. doi:10.1172/ JCI28123.

13. Goukassian, D.A., et al. 2007. Tumor necrosis factor- alpha receptor $\mathrm{p} 75$ is required in ischemia-induced neovascularization. Circulation. 115:752-762.

14. Ratcliffe, P.J. 2007. HIF-1 and HIF-2: working alone or together in hypoxia? J. Clin. Invest. 117:862-865. doi:10.1172/JCI31750.

15. Forsythe, J.A., et al. 1996. Activation of vascular endothelial growth factor gene transcription by hypoxia-inducible factor $1 . \mathrm{Mol}$. Cell. Biol. 16:4604-4613

16. Shi, S., and Gronthos, S. 2003. Perivascular niche of postnatal mesenchymal stem cells in human bone marrow and dental pulp. J. Bone Miner. Res. 18:696-704.

17. Farrington-Rock, C., et al. 2004. Chondrogenic and adipogenic potential of microvascular pericytes. Circulation. 110:2226-2232.

18. Tintut, Y., et al. 2003. Multilineage potential of cells from the artery wall. Circulation. 108:2505-2510.

19. Schor, A.M., Allen, T.D., Canfield, A.E., Sloan, P., and Schor, S.L. 1990. Pericytes derived from the retinal microvasculature undergo calcification in vitro. J. Cell Sci. 97:449-461.

20. Brunelli, S., et al. 2004. Msx2 and necdin combined activities are required for smooth muscle differentiation in mesoangioblast stem cells. Circ. Res. 94:1571-1578

21. Shao, J.S., et al. 2005. Msx2 promotes cardiovascular calcification by activating paracrine Wn signals. J. Clin. Invest. 115:1210-1220. doi:10.1172/ JCI200524140.

22. Cheng, S.L., Shao, J.S., Charlton-Kachigian, N Loewy, A.P., and Towler, D.A. 2003. MSX2 promotes osteogenesis and suppresses adipogenic differentiation of multipotent mesenchymal progenitors. J. Biol. Chem. 278:45969-45977.

23. Sakurai, H., et al. 2006. In vitro modeling of par- axial and lateral mesoderm differentiation reveals early reversibility. Stem Cells. 24:575-586.

24. Kalajzic, I., et al. 2006. Myofibroblast/pericyte phenotype of the osteoprogenitor cell. J. Bone Miner. Res. 21:S2, abstract 1004.

25. Eames, B.F., de la Fuente, L., and Helms, J.A. 2003. Molecular ontogeny of the skeleton. Birth Defects Res. C Embryo Today. 69:93-101.

26. Burrows, A.M., et al. 2001. Endocranial vascular patterns in a familial rabbit model of coronal suture synostosis. Cleft Palate Craniofac. J. 38:615-621.

27. Henderson, J.H., Longaker, M.T., and Carter, D.R. 2004. Sutural bone deposition rate and strain magnitude during cranial development. Bone. 34:271-280.

28. Fong, K.D., et al. 2003. Mechanical strain affects dura mater biological processes: implications for mmature calvarial healing. Plast. Reconstr. Surg. 112:1312-1327

29. Carvalho, R.S., et al. 2004. The role of angiogenesis in a murine tibial model of distraction osteogenesis. Bone. 34:849-861.

30. George, D.J., and Kaelin, W.G., Jr. 2003. The von Hippel-Lindau protein, vascular endothelial growth factor, and kidney cancer. N. Engl. J. Med. 349:419-421.

31. Esner, M., et al. 2006. Smooth muscle of the dorsal aorta shares a common clonal origin with skeletal muscle of the myotome. Development. 133:737-749

32. Tagliafico, E., et al. 2004. TGFbeta/BMP activate the smooth muscle/bone differentiation programs in mesoangioblasts. J. Cell Sci. 117:4377-4388.

33. Cossu, G., and Bianco, P. 2003. Mesoangioblasts - vascular progenitors for extravascular mesodermal tissues. Curr. Opin. Genet. Dev. 13:537-542.

\title{
Sizing up sialic acid in glomerular disease
}

\author{
Susan E. Quaggin \\ The Samuel Lunenfeld Research Institute, Mount Sinai Hospital, and Division of Nephrology, St. Michael's Hospital, \\ University of Toronto, Toronto, Ontario, Canada.
}

\begin{abstract}
A new study by Galeano and colleagues in this issue of the JCI reports the first glomerular disease caused by a genetic defect in sialic acid biosynthesis (see the related article beginning on page 1585). Mice that harbor mutations in the Gne/Mnk gene produce lower amounts of sialic acid, suffer from hematuria, proteinuria, and structural defects in the glomerulus and die within days after birth. Remarkably, the lesion can be reversed through dietary addition of $N$-acetylmannosamine, a sialic acid precursor, raising the intriguing possibility that this approach might have therapeutic benefit in patients with glomerular disease.
\end{abstract}

In this issue of the JCI, Galeano, Huizing, and colleagues (1) describe kidney defects in knockin mice that harbor the M712T muta-

Nonstandard abbreviations used: Gne, uridine diphospho- $\mathrm{N}$-acetylglucosamine 2-epimerase; HIBM, hereditary inclusion body myopathy; ManNAc, $N$-acetylmannosamine; Mnk, ManNAc kinase; PC, podocalyxin; SD, slit diaphragm.

Conflict of interest: The author is the recipient of a research grant from Genzyme and is a scientific consultant for Genentech.

Citation for this article: J. Clin. Invest. 117:1480-1483 (2007). doi:10.1172/JCI32482. tion in the gene encoding the key bifunctional enzyme of sialic acid biosynthesis - uridine diphospho- $N$-acetylglucosamine 2-epimerase/ $\mathrm{N}$-acetylmannosamine (ManNAc) kinase (GNE/MNK) (Gne $e^{M 712 T / M 712 T}$ mice) $(2,3)$. In patients, mutations in the GNE gene result in the autosomal recessive neuromuscular disorder, hereditary inclusion body myopathy (HIBM; MIM 600737) that presents late in life as a slowly progressive myopathy (4, 5). The $N$-acetylglucosamine/ ManNAc enzyme is ubiquitously expressed and catalyzes the first rate-limiting steps in the biosynthesis of sialic acid (Figure 1). $5-\mathrm{N}$-acetylneuraminic acid (Neu5Ac) is the most plentiful mammalian sialic acid and is the terminal sugar on glycoconjugates, where it functions in cellular interactions and signaling. Muscle fibers from patients with HIBM exhibit reduced sialylation of proteins, which is believed to underlie disease pathogenesis. Currently, there is no effective therapy for this disorder.

In the current study (1), the investigators sought to develop a mouse model to test whether dietary supplementation of sialic acid or its precursor, ManNAc, could reverse the hyposialylation defect observed in patients. Standard Gne knockout mice die in utero (6). To overcome this early mortality, the authors generated a mouse carrying one of the most common nonlethal mutations observed in patients with HIBM. Surprisingly, mice homozygous for 